ECIPERÚ

\title{
ESTUDIO PRELIMINAR DE LA ACTIVIDAD ANTIINFLAMATORIA DEL EXTRACTO ETANOLICO DE LOS TALLOS DE Ageratina sternbergiana (DC.) R.M. King \& H.Rob "Zun Zun"
}

\author{
Narciso León S. ${ }^{1}$ Luis Felix V. ${ }^{1}$, Juana Chávez F. ${ }^{1}$, Paola Quispe F. ${ }^{2}$ \\ Docentes $^{1}$ y alumna ${ }^{2}$ de la Facultad de Farmacia y Bioquímica, Universidad Wiener. \\ Av. Arequipa 440 - Lima
}

\section{Resumen}

La presente investigación se llevó a cabo con el objeto de determinar científicamente la propiedad antiinflamatoria atribuida popularmente a la planta Ageratina sternbergiana (DC.) R.M. King \& H.Rob "Zun Zun", utilizada para afecciones que implican procesos inflamatorios. Se evaluó la actividad antiinflamatoria mediante el Método del edema subplantar según Winter et. al. El método consiste en provocar una reacción inflamatoria en la pata posterior izquierda de los animales de experimentación, que puede ser reducida por las sustancias antiinflamatorias. Se utilizaron 18 ratones albinos (hembras y machos) cepa Balb C53 distribuidos en 6 grupos: control (sin tratamiento) ,3 grupos con extracto etanólico de tallos a dosis de $50 \mathrm{mg} / \mathrm{Kg}, 100 \mathrm{mg}$ / Kg, 200 mg / Kg; 1 grupo con extracto etanólico de tallos hidrolizado a dosis de $160 \mathrm{mg} / \mathrm{Kg}$ y 1 grupo con indometacina en dosis de $10 \mathrm{mg} / \mathrm{Kg}$. La aplicación de todos los tratamientos fue por vía oral. Los resultados farmacológicos indican que el tratamiento con extracto etanólico de los tallos de Ageratina sternbergiana (DC.) R.M. King \& H.Rob "Zun Zun", a dosis de $200 \mathrm{mg} / \mathrm{Kg}$ es el que tiene mayor efectividad antiinflamatoria. Se realizó el análisis fitoquímico de los tallos de la planta, la cual reveló la presencia de azúcares, flavonoides, alcaloides, esteroides, cumarinas, taninos y aminoácidos.

Palabras claves: Ageratina sternbergiana, actividad antiinflamatoria, método del edema subplantar, análisis fitoquímico.

\begin{abstract}
This research was conducted for determine scientifically the anti-inflammatory property attributed popularly to the plant Ageratina sternbergiana (DC.) RM King \& H. Rob "Zun Zun", used for inflammation. The Antiinflammatory activity was evaluated by subplantar edema method according to Winter et . al. The method consist to cause inflammation in the left hind paw of the experimental animals, which can be reduced by the anti-inflammatory substances. We used 18 albino mice (male and female) strain Balb C53 in 6 groups: control (no treatment), 3 groups with ethanol extract of stems at doses of $50 \mathrm{mg} / \mathrm{kg}, 100 \mathrm{mg} / \mathrm{kg}, 200 \mathrm{mg} / \mathrm{kg}$ and 1 group hydrolyzed ethanol extract of stems at doses of $160 \mathrm{mg} / \mathrm{kg}$ and 1 group with indomethacin in doses of $10 \mathrm{mg} / \mathrm{kg}$. The application of all treatments were oral. The pharmacological results indicate that treatment with ethanol extract of the stems of Ageratina sternbergiana (DC.) RM King \& H. Rob "Zun Zun", at doses of $200 \mathrm{mg} / \mathrm{kg}$ is the one most effective anti-inflammatory. Phytochemical analysis was performed of the stems of the plant, which revealed the presence of sugars, flavonoids, alkaloids, steroids, coumarins, tannins and amino acids.
\end{abstract}

Keywords: Ageratina sternbergiana, inflammatory activity, subplantar edema method, phytochemical analysis, flavonoids.

\section{Introducción}

La flora peruana alberga innumerables especies vegetales con propiedades curativas, entre las que se encuentran plantas con efecto antiinflamatorio. Una de ellas es la Ageratina sternbergiana (DC.) R.M. King \& H.Rob "Zun Zun", una especie endémica del Perú, a la que se atribuye propiedades antiinflamatorias y de la cual se conocen los siguientes nombres comunes: Manka p'aki ("Manca paqui"), en el Cuzco'; Lambrakaña macho y Lambrakaña hembra, en el sur andino ${ }^{2}$; la cual es comúnmente empleada como agua de tiempo. 
Es preciso destacar que se han encontrado algunos reportes sobre el estudio de las flavanonas ${ }^{3,4}$ (un tipo de flavonoide) que contiene dicha especie, pero muy poco sobre estudios fitoquímicos y farmacológicos.

Por las razones antes mencionadas se considera a la Ageratina sternbergiana (DC.) R.M. King \& H.Rob "Zun Zun" para el presente estudio. Para ello se ensayó con el extracto etanólico a diferentes concentraciones con el propósito de determinar el uso popular de esta especie medicinal como antiinflamatoria. Los objetivos específicos fueron identificar los metabolitos primarios y secundarios presentes en el extracto etanólico de los tallos de Ageratina sternbergiana (DC.) R.M. King \& H.Rob "Zun Zun" y determinar el efecto antiinflamatorio del extracto etanólico de los tallos de Ageratina sternbergiana (DC.) R.M. King \& H.Rob "Zun Zun" en animales de experimentación.

\section{Materiales Y Métodos}

1. Preparación del extracto etanólico para el estudio fitoquímico

1.1. Recolección: se recolectó 3 kilos de tallos de la especie Ageratina sternbergiana "Zun Zun"en el mes de Enero del 2010 en el distrito de Cachicadán, provincia de Santiago de Chuco departamento de La Libertad.

1.2. Desecación: los tallos se desecaron en estufa a $40^{\circ} \mathrm{C}$.

1.3. Molienda: se utilizó molino de cuchillas Willey Hill St. Model $\mathrm{N}^{\circ} 3$, obteniéndose un polvo fino.

1.4. Preparación del extracto etanólico: Se pesó $500 \mathrm{~g}$ del polvo seco; se añadió 3 litros de etanol $70 \%$ y se maceró en frasco color ámbar por 7 días con agitación diaria. Posteriormente se filtró con papel filtro y gasa; el líquido filtrado fue concentrado en el rota vapor (Buchi R 210), llevado a sequedad a la estufa ((Memmert $\circledR) \quad 40^{\circ} \mathrm{C}$ obteniéndose el extracto seco.

\section{Ensayos preliminares}

2.1. Prueba de solubilidad: el extracto

\footnotetext{
etanólico se sometió a pruebas de
solubilidad con solventes de diferentes

etanólico se sometió a pruebas de
solubilidad con solventes de diferentes
}

polaridades. En tubos de ensayo se colocó una pequeña porción del extracto etanólico se le agregó $1 \mathrm{~mL}$ del solvente respectivo benceno, acetona, n- butanol, etanol, metanol, agua destilada, n-hexano, acetato de etilo, cloroformo, éter etílico, éter de petróleo , se agitó y se observaron los resultados.

2.2. Marcha fitoquímica: Las reacciones de identificación se efectuaron mediante técnicas reportadas por $\operatorname{Lock}^{5}$ y Domínguez ${ }^{6}$. El extracto seco se solubilizó en etanol y se realizaron ensayos para detectar los metabolitos primarios y secundarios como compuestos fenólicos, flavonoides, taninos, aminoácidos, carbohidratos, esteroides y cumarinas

\section{Hidrólisis de Flavonoides ${ }^{7}$}

Con el objetivo de aislar flavonoides, se mezcló $1 \mathrm{~g}$ de extracto etanólico de los tallos de Ageratina sternbergiana (DC.) R.M. King \& H.Rob "Zun Zun", con 10 $\mathrm{mL}$ de metanol por 5 minutos en un baño de agua $\left(60^{\circ} \mathrm{C}\right)$.Luego se tomó $5 \mathrm{ml}$ de la solución y se concentró hasta obtener 2 $\mathrm{mL}$. Posteriormente se adicionó $1 \mathrm{~mL}$ de agua y $10 \mathrm{ml}$ de acetato de etilo, y se agitó por 10 minutos. Finalmente se separó la fase orgánica y se concentró hasta obtener un volumen de $1 \mathrm{~mL}$.

\section{Estudio Farmacologico ${ }^{8}$}

Para determinar el efecto antiinflamatorio de Ageratina sternbergiana (DC.) R.M. King \& H.Rob "Zun Zun" se utilizó: El Método del edema subplantar según Winter et. al. Este método consiste en la administración subcutánea de albúmina $1 \%$ a nivel de la aponeurosis plantar del ratón, provocando una reacción de carácter inflamatorio mediada por liberación de diversos autacoides (histamina, serotonina, bradiquinina, prostaglandinas fundamentalmente PGEI, PGE2, PGF2alfa, etc), la extravasación de proteínas, tiene lugar durante toda la respuesta. 


\section{Evaluación de la Actividad Antiinflamatoria}

\section{Distribución de la muestra:}

En los ensayos se utilizaron 6 grupos distribuidos en forma aleatoria.

Los grupos fueron formados de la siguiente manera:

El grupo 1: extracto etanólico de tallos de "Zun Zun", 50 mg / Kg

El grupo 2: extracto etanólico de tallos de "Zun Zun", 100 mg / Kg

El grupo 3: extracto etanólico de tallos de "Zun Zun", 200 mg / Kg

El grupo 4: recibió Indometacina $10 \mathrm{mg} / \mathrm{kg}$ (control positivo)

El grupo 5: extracto hidrolizado de tallos de "Zun Zun", 160 mg / Kg

El grupo 6: recibió agua destilada (control negativo)

\section{Procedimiento:}

Los animales fueron privados de alimentos 24 horas antes del ensayo y tuvieron acceso libre al agua.

La administración de los extractos y la droga testigo fue por vía oral a través de una sonda orogástrica; como droga testigo se empleo la Indometacina, en dosis de
$10 \mathrm{mg} / \mathrm{Kg}, 1$ hora después se inyectó $0,1 \mathrm{~mL}$ de solución de albumina al $1 \%$ en la aponeurosis de la pata izquierda de cada animal.

\section{Técnica de medición:}

Tres horas después de la aplicación de la solución de albumina al $1 \%$ se sacrifican a los animales de experimentación; ratones albinos (hembras y machos) cepa Balb C53 por dislocación cervical y luego se procede a cortar ambas patas utilizando una tijera, estas porciones son pesadas inmediatamente y por separado, tomando nota de los respectivos pesos.

De cada peso en gramos de la patas izquierda y derecha, se comparan los resultados y se sacan promedios para analizarlos en las graficas.

\section{Resultados}

\section{Estudio fitoquímico:}

La prueba de solubilidad del extracto etanólico "Zun Zun" se muestra en la tabla 1. Por su solubilidad en metanol y etanol, se deduce que los componentes químicos mayoritarios son de naturaleza polar ${ }^{5}$.

Tabla 1. Prueba de solubilidad del extracto etanólico de tallos de Ageratina sternbergiana (DC.) R.M. King \& H.Rob "Zun Zun"

\begin{tabular}{lc}
\hline \multicolumn{1}{c}{ Solvente } & Solubilidad \\
\hline Agua destilada & +++ \\
Etanol & +++ \\
Metanol & +++ \\
n-Butanol & +- \\
Acetato de etilo & - \\
n-Hexano & - \\
Cloroformo & +- \\
Benceno & +- \\
Acetona & - \\
Éter etílico & - \\
Éter de petróleo & - \\
\hline
\end{tabular}

Leyenda: Muy soluble: +++

Ligeramente soluble: + -

Insoluble: -

La marcha fitoquímica permite determinar cualitativamente los principales grupos de constituyentes químicos de la planta. En la tabla 2 se muestra los resultados de la marcha fitoquímica de la especie Ageratina sternbergiana "Zun Zun". 
Tabla 2. Marcha Fitoquímica del extracto etanólico de de tallos de Ageratina sternbergiana (DC.) R.M. King \& H.Rob "Zun Zun"

\begin{tabular}{lll}
\hline Reactivo & $\begin{array}{l}\text { Metabolito primario } \\
\text { y secundario }\end{array}$ & Resultado \\
\hline $\begin{array}{l}\text { Tricloruro de aluminio } 1 \% \\
\text { Shinoda }\end{array}$ & $\begin{array}{l}\text { Flavonoides } \\
\text { Flavonoides }\end{array}$ & + \\
\hline
\end{tabular}

\begin{tabular}{|c|c|c|c|c|c|}
\hline $\mathrm{N}^{\circ}$ & RATON & $\begin{array}{l}\text { PATA DERECHA SIN } \\
\text { ALBUMINA }\end{array}$ & $\begin{array}{l}\text { PATA } \\
\text { CON ALB }\end{array}$ & $\begin{array}{l}\text { IZQUIERDA } \\
\text { 3UMINA }\end{array}$ & PESO/ANIMAL \\
\hline 1 & RATON COLA ROJA & $0,18 \mathrm{~g}$ & $0,26 \mathrm{~g}$ & & $28,6 \mathrm{~g}$ \\
\hline 2 & RATON COLA AZUL & $0,18 \mathrm{~g}$ & $0,27 \mathrm{~g}$ & & $29,6 \mathrm{~g}$ \\
\hline \multirow[t]{15}{*}{3} & $\begin{array}{ll}\text { RATON } & \text { COLA } \\
\text { NEGRA } & \\
\end{array}$ & $0,19 \mathrm{~g}$ & $0,21 \mathrm{~g}$ & & $39,2 \mathrm{~g}$ \\
\hline & PROMEDIO & $0,18 \mathrm{~g}$ & $0,25 \mathrm{~g}$ & & $32,5 \mathrm{~g}$ \\
\hline & Tricloruro férrico $1 \%$ & \multicolumn{2}{|c|}{ Compuestos fenólicos } & + & \\
\hline & Gelatina - NaOH $1 \%$ & Taninos & & + & \\
\hline & Molish & Carbohidratos & & + & \\
\hline & $\mathrm{NaOH} 5 \%$ & Cumarina & & + & \\
\hline & Dragendorff & \multirow{2}{*}{ Alcaloides } & & + & \\
\hline & Mayer & & & & \\
\hline & Popoff & Alcaloides & & + & \\
\hline & Wagner & Alcaloides & & + & \\
\hline & Liebermann - Burchard & \multirow{2}{*}{$\begin{array}{l}\text { Esteroides } \\
\text { Esteroides }\end{array}$} & & \multirow[t]{2}{*}{+} & \\
\hline & Salkowski & & & & \\
\hline & Fehling A, B & & & + & \\
\hline & Ninhidrina $1 \%$ & \multicolumn{2}{|c|}{ Grupo amino libre } & \multirow[t]{2}{*}{+} & \\
\hline & Índice afrosimétrico & \multicolumn{2}{|c|}{ Saponinas esteroidales } & & \\
\hline
\end{tabular}

Leyenda: ( - ) ausencia ( + ) presencia

\section{Estudio Farmacológico:}

Tabla 3: Cuadro estadístico de las diferencias de los pesos de las patas inflamadas y las normales de los ratones del grupo con extracto etanólico de tallos de "zun zun", $50 \mathrm{mg} / \mathrm{kg}$

\section{GRUPO No 1}

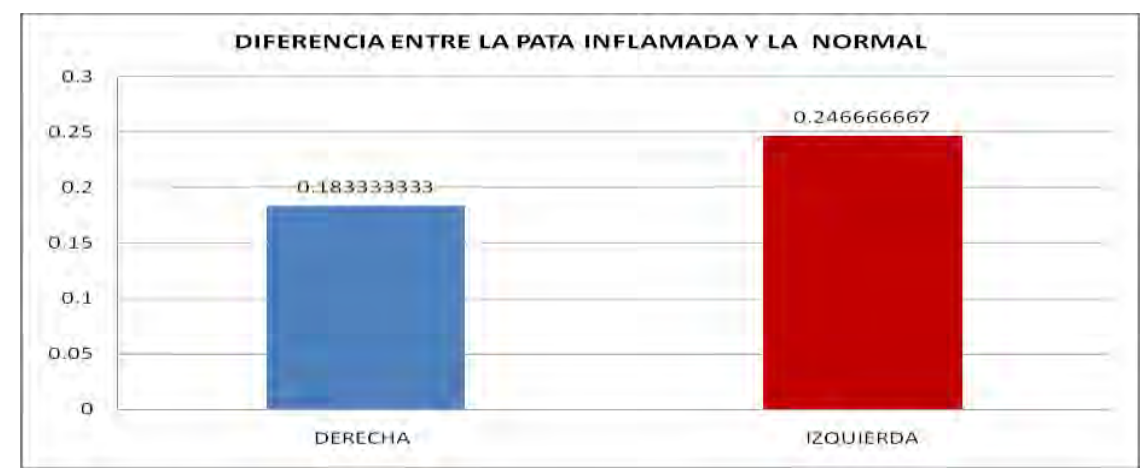

Figura $N^{\circ}$ 1: Diferencia de los pesos promedios de las patas de los ratones del grupo con extracto etanólico de tallos de "Zun Zun", 50 mg / kg. 
Tabla 4: Cuadro estadístico de las diferencias de los pesos de las patas inflamadas y las normales de los ratones del grupo con extracto etanólico de tallos de "zun zun", $100 \mathrm{mg} / \mathrm{kg}$

\begin{tabular}{|l|l|l|l|l|}
\hline$N^{\circ}$ & RATON & $\begin{array}{l}\text { PATA DERECHA SIN } \\
\text { ALBUMINA }\end{array}$ & $\begin{array}{l}\text { PATA IZQUIERDA } \\
\text { CON ALBUMINA }\end{array}$ & PESO/ANIMAL \\
\hline 1 & RATON COLA ROJA & $0,18 \mathrm{~g}$ & $0,21 \mathrm{~g}$ & $30,2 \mathrm{~g}$ \\
\hline 2 & RATON COLA AZUL & $0,17 \mathrm{~g}$ & $0,22 \mathrm{~g}$ & $33,4 \mathrm{~g}$ \\
\hline 3 & $\begin{array}{l}\text { RATON COLA } \\
\text { NEGRA }\end{array}$ & $0,18 \mathrm{~g}$ & $0,21 \mathrm{~g}$ & $28,1 \mathrm{~g}$ \\
\hline & PROMEDIO & $0,18 \mathrm{~g}$ & $0,21 \mathrm{~g}$ & $30,6 \mathrm{~g}$ \\
\hline
\end{tabular}

GRUPO No 2

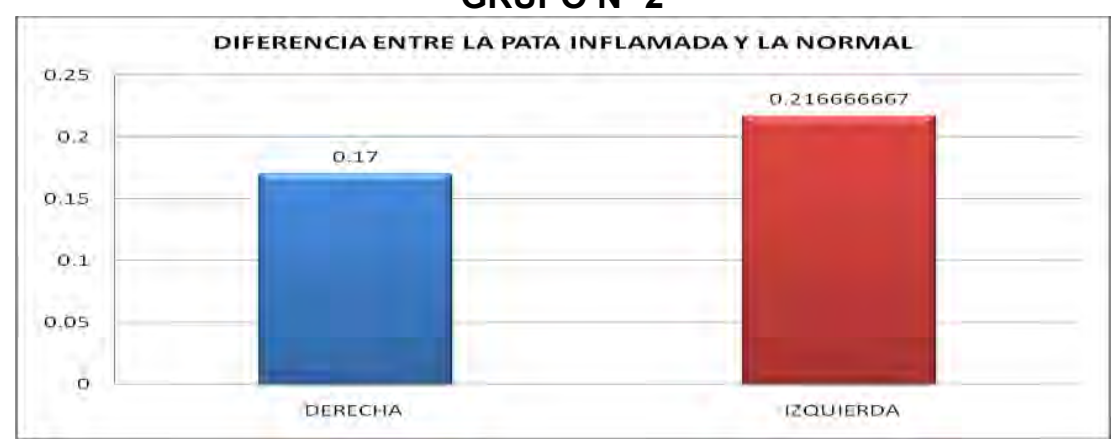

Figura N²: Diferencia de los pesos promedios de las patas de los ratones del grupo con extracto etanólico de tallos de "Zun Zun", $100 \mathrm{mg} / \mathrm{kg}$.

Tabla 5: Cuadro estadístico de las diferencias de los pesos de las patas inflamadas y las normales de los ratones del grupo con extracto etanólico de tallos de "zun zun", $200 \mathrm{mg} / \mathrm{kg}$

\begin{tabular}{|l|l|l|l|l|}
\hline$N^{\circ}$ & RATON & $\begin{array}{l}\text { PATA DERECHA SIN } \\
\text { ALBUMINA }\end{array}$ & $\begin{array}{l}\text { PATA IZQUIERDA } \\
\text { CON ALBUMINA }\end{array}$ & PESO/ANIMAL \\
\hline 1 & RATON COLA ROJA & $0,17 \mathrm{~g}$ & $0,25 \mathrm{~g}$ & $27 \mathrm{~g}$ \\
\hline 2 & RATON COLA AZUL & $0,16 \mathrm{~g}$ & $0,19 \mathrm{~g}$ & $26 \mathrm{~g}$ \\
\hline 3 & $\begin{array}{l}\text { RATON } \\
\text { COLA NEGRA }\end{array}$ & $0,18 \mathrm{~g}$ & $0,21 \mathrm{~g}$ & $37,8 \mathrm{~g}$ \\
\hline & PROMEDIO & $0,17 \mathrm{~g}$ & $0,22 \mathrm{~g}$ & $30,27 \mathrm{~g}$ \\
\hline
\end{tabular}

GRUPO N ${ }^{\circ} 3$

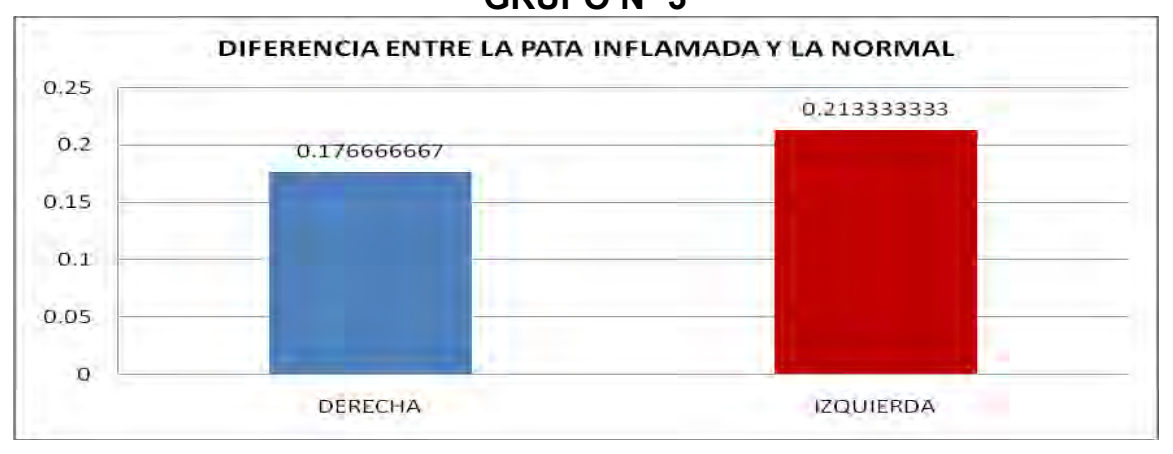

Figura №3: Diferencia de los pesos promedios de las patas de los ratones del grupo con extracto etanólico de tallos de "Zun Zun", 200 mg / kg. 
Tabla 6: Cuadro estadístico de las diferencias de los pesos de las patas inflamadas y las normales de los ratones del grupo que recibió indometacina $10 \mathrm{mg} / \mathrm{kg}$ (control positivo)

\begin{tabular}{|l|l|l|l|l|}
\hline \multicolumn{1}{|c|}{$\mathrm{N}^{\circ}$} & RATON & $\begin{array}{l}\text { PATA DERECHA SIN } \\
\text { ALBUMINA }\end{array}$ & $\begin{array}{l}\text { PATA IZQUIERDA } \\
\text { CON ALBUMINA }\end{array}$ & PESO/ANIMAL \\
\hline 1 & RATON COLA ROJA & $0,16 \mathrm{~g}$ & $0,20 \mathrm{~g}$ & $26,5 \mathrm{~g}$ \\
\hline 2 & RATON COLA AZUL & $0,18 \mathrm{~g}$ & $0,21 \mathrm{~g}$ & $26,7 \mathrm{~g}$ \\
\hline 3 & $\begin{array}{l}\text { RATON COLA } \\
\text { NEGRO } 0,20 \mathrm{~g}\end{array}$ & $0,28 \mathrm{~g}$ & $35 \mathrm{~g}$ \\
\hline & PROMEDIO & $0,18 \mathrm{~g}$ & $0,23 \mathrm{~g}$ & $29,4 \mathrm{~g}$ \\
\hline
\end{tabular}

GRUPO No 4

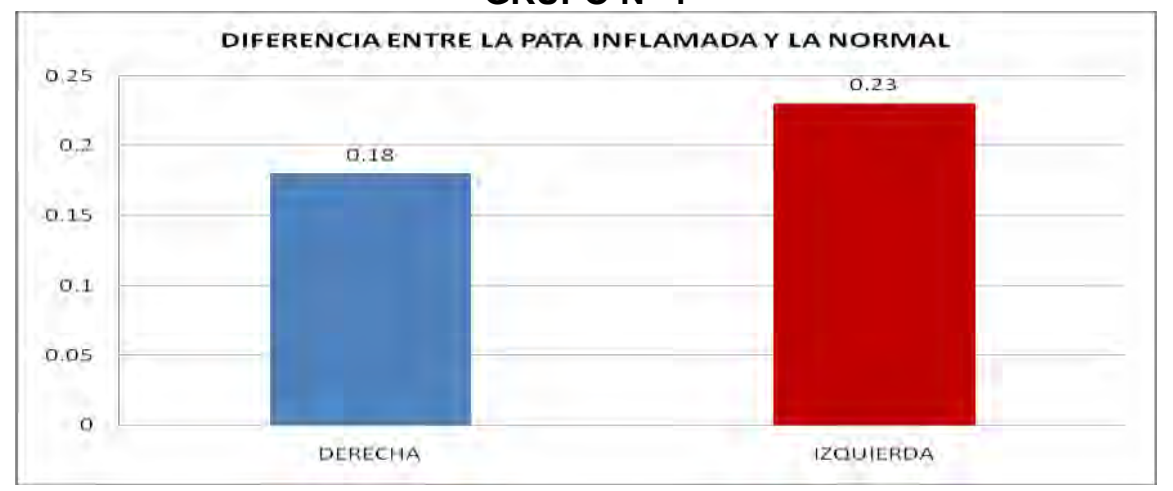

Figura №4: Diferencia de los pesos promedios de las patas de los ratones del grupo que recibió indometacina $10 \mathrm{mg} / \mathrm{kg}$ (control positivo).

Tabla 7: Cuadro estadístico de las diferencias de los pesos de las patas inflamadas y las normales de los ratones del grupo que recibió extracto hidrolizado de tallos de "zun zun", $160 \mathrm{mg} / \mathrm{kg}$

\begin{tabular}{|l|l|l|l|l|}
\hline$N^{\circ}$ & RATON & $\begin{array}{l}\text { PATA DERECHA SIN } \\
\text { ALBUMINA }\end{array}$ & $\begin{array}{l}\text { PATA IZQUIERDA } \\
\text { CON ALBUMINA }\end{array}$ & PESO/ANIMAL \\
\hline 1 & RATON COLA ROJA & $0,20 \mathrm{~g}$ & $0,28 \mathrm{~g}$ & $35,3 \mathrm{~g}$ \\
\hline 2 & RATON COLA AZUL & $0,16 \mathrm{~g}$ & $0,21 \mathrm{~g}$ & $26,9 \mathrm{~g}$ \\
\hline 3 & $\begin{array}{l}\text { RATON COLA } \\
\text { NEGRA }\end{array}$ & $0,18 \mathrm{~g}$ & $0,25 \mathrm{~g}$ & $26,9 \mathrm{~g}$ \\
\hline & PROMEDIO & $0,18 \mathrm{~g}$ & $0,25 \mathrm{~g}$ & $29,7 \mathrm{~g}$ \\
\hline
\end{tabular}

\section{GRUPO No 5}

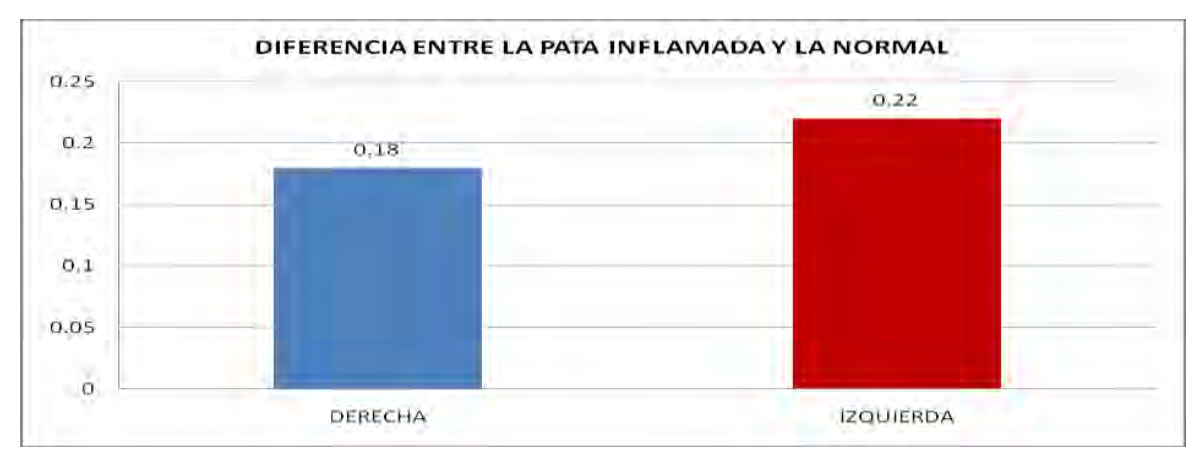

Figura №5: Diferencia de los pesos promedios de las patas de los ratones del grupo que recibió extracto hidrolizado de tallos de "ZUN ZUN", $160 \mathrm{mg} / \mathrm{kg}$. 
Tabla 8: Cuadro estadístico de las diferencias de los pesos de las patas inflamadas y las normales de los ratones del grupo control

\begin{tabular}{|l|l|l|l|l|}
\hline$N^{\circ}$ & RATON & $\begin{array}{l}\text { PATA DERECHA SIN } \\
\text { ALBUMINA }\end{array}$ & $\begin{array}{l}\text { PATA IZQUIERDA } \\
\text { CON ALBUMINA }\end{array}$ & PESO/ANIMAL \\
\hline 1 & RATON COLA ROJA & $0,17 \mathrm{~g}$ & $0,21 \mathrm{~g}$ & $30,5 \mathrm{~g}$ \\
\hline 2 & RATON COLA AZUL & $0,20 \mathrm{~g}$ & $0,23 \mathrm{~g}$ & $30,6 \mathrm{~g}$ \\
\hline 3 & $\begin{array}{l}\text { RATON COLA } \\
\text { NEGRA }\end{array}$ & $0,17 \mathrm{~g}$ & $0,22 \mathrm{~g}$ & $31,8 \mathrm{~g}$ \\
\hline & PROMEDIO & $0,18 \mathrm{~g}$ & $0,22 \mathrm{~g}$ & $31 \mathrm{~g}$ \\
\hline
\end{tabular}

GRUPO N 6

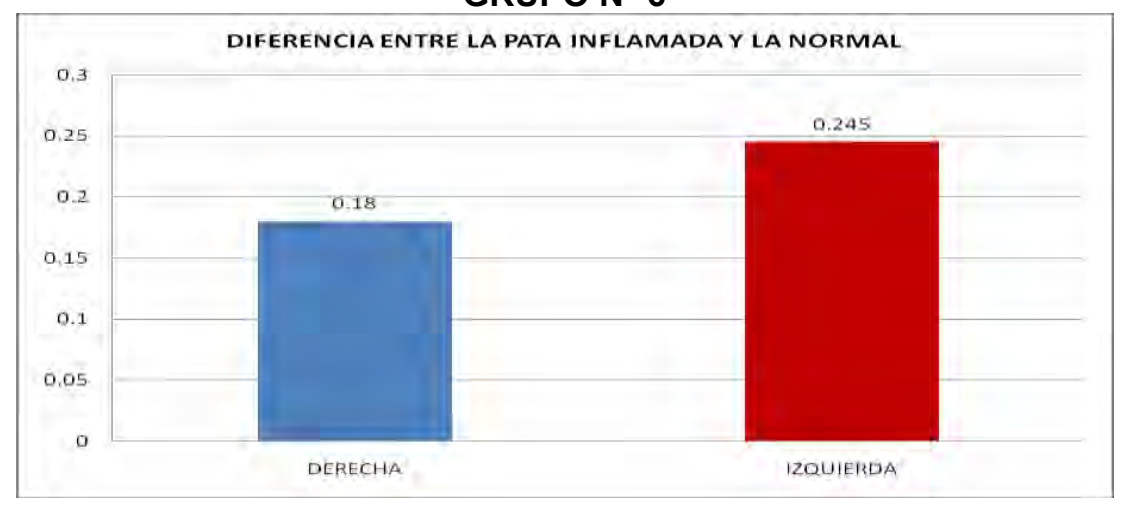

Figura Nº6: Diferencia de los pesos promedios de las patas de los ratones del grupo control.

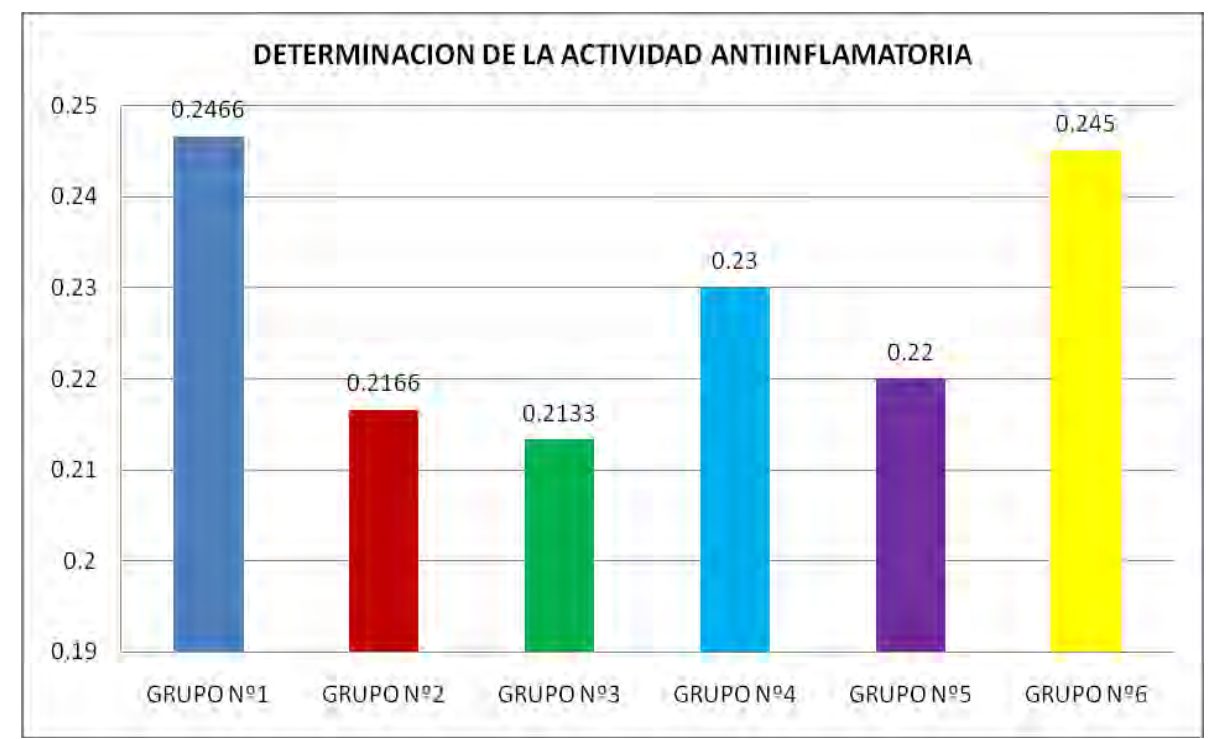

Figura Nº7: Determinación de la Actividad Antiinflamatoria de cada grupo de estudio. 


\section{Discusión}

La prueba de solubilidad del extracto etanólico "Zun Zun" se muestra en la tabla 1. Se observa su solubilidad en etanol, metanol y agua destilada; de donde se deduce que los componentes químicos mayoritarios son de naturaleza y estructura polar ${ }^{5}$.

En el análisis fitoquímico encontramos gran cantidad de flavonoides, al igual que azúcares, aminoácidos, esteroides, alcaloides y cumarinas, por lo que asumimos que la presencia de estos metabolitos primarios y secundarios podría explicar la acción farmacológica y justificar el empleo de la planta en el tratamiento de afecciones inflamatorias.

El extracto etanólico de los tallos de Ageratina sternbergiana (DC.) R.M. King \& H.Rob "Zun Zun", tras la evaluación farmacológica evidencia respuesta a la acción irritante de la albumina 1\% validando así el efecto farmacológico atribuido a la planta, como se observa en la figura $\mathrm{N}^{\circ} 7$.

La actividad antiinflamatoria se comparó con un producto farmacéutico Indometacina $25 \mathrm{mg}$, obteniendo una mejor respuesta en el extracto etanólico a mayor dosis $(200 \mathrm{mg} / \mathrm{Kg})$ como se observa en la figura $\mathrm{N}^{\circ} 7$.

En los datos obtenidos según se muestra (tabla 5 y 7) y (figura $N^{\circ} 7$ ) indican que existe mayor acción farmacológica con el extracto a mayor dosis que con el extracto hidrolizado, se entiende que posiblemente el flavonoide para que tenga mayor acción debe estar acompañado de los metabolitos primaros o secundarios de la planta.

\section{Conclusiones}

- Se identificó los metabolitos primarios y secundarios presentes en el extracto etanólico de los tallos de Ageratina sternbergiana (DC.) R.M. King \& H.Rob "Zun Zun", los cuales son solubles en solventes polares.

- Se determinó que el efecto antiinflamatorio del extracto etanólico de los tallos de Ageratina sternbergiana (DC.) R.M. King \& H.Rob "Zun Zun" a dosis de $200 \mathrm{mg} / \mathrm{Kg}$ es el que evidencia una mejor respuesta antiinflamatoria en animales de experimentación.

\section{Agradecimientos}

Nuestro agradecimiento a María Crespina Pérez Castillo pobladora del distrito de Cachicadán, provincia de Santiago de Chuco departamento de La Libertad por brindarnos la muestra vegetal.

\section{REFERENCIAS BIBLIOGRÁFICAS}

1. Registro botánico de la Asociación Pukllasunchis. La Microcuenca del Río Kachimayu Flora y Fauna de la Región del Cusco. Acceso: 16/11/2010. Disponible en: http://zambolandia.com/papers/puklla1/reg_b ot_flores.html

2. Ayala D. César L. Las Plantas Medicinales en el Sur Andino del Perú Acceso: 16/11/2010. Disponible http://esmiperu.blogspot.com/2007/05/lasplantas-medicinales-en-el-sur.html

3. Antonio G. Gonzales, Braulio M. Fraga, Victor P. Garcia y Melchor G. Hernandez. Eupatarone. A 2 - Acetylbenzofuran from Eupatorium Sternbergianum .Rev. Phytochemistry 1982 .Vol 21, № 7, pp. $1826-$ 1827.

4. Antonio G. Gonzales, Braulio M. Fraga, Victor P. Garcia y Melchor G. Hernandez. Sternbina, Una Nueva Flavanona del Eupatorium Sternbergianum. Rev. Latinoamericana de Química 1984. 14-3. 115-117.

5. Lock de Ugaz, O. Investigación fitoquímica. Segunda Edición .Editorial PUCP.Lima.1994

6. Domínguez, X. Métodos de Investigación Fitoquímica. Editorial Limusa. Mexico. Segunda Edición.1979.

7. Aragadvay Yungán Sandra Piedad. Elaboración y Control de Calidad de Tintura y Gel Cicatrizante y Antiinflamatorio a Base de Chilca (Baccharis latifolia) y Hierbamora (Solanum nigrum). Acceso: 14/08/2010. Disponible en: www.dspace.espoch.edu.ec/bitstream/123456 789/216/1/56T00190.pdf

8. Castañeda, C.B.; Manrique M.R.; Ibáñez V.L. Evaluación del Efecto Antiinflamatorio del Extracto Acuoso de las Semillas de Lupinus Mutabilis Sweet (Tarwi, Chocho), en Animales de Experimentación. Acceso: 10/11/2010. Disponible en: www.usmp.edu.pe/medicina/horizonte/2002/Art 3_Vol2_N1-2.pdf

E-mail: melissa_1418@hotmail.com 